\title{
SELEÇÃO DE UMA LÂMPADA DE FILAMENTO EM UMA EMPRESA DE ILUMINAÇÃO DECORATIVA: UMA APLICAÇÃO DO MÉTODO MULTICRITÉRIO SAPEVO-M
}

\author{
Camila Guimarães Monteiro de Freitas Alves \\ Universidade Federal Fluminense (UFF) / Instituto Federal de Educação, Ciência e \\ Tecnologia do Rio de Janeiro (IFRJ) \\ Rua Passo da Pátria, 156 - 209, São Domingos, Niterói - RJ / Rua José Breve, 550, Centro, \\ Pinheiral - RJ \\ camila_gmf@hotmail.com \\ Marcos dos Santos \\ Centro de Análises de Sistemas Navais (CASNAV) / Instituto Militar de Engenharia (IME) \\ Praça Barão de Ladário, Ilha das Cobras, Rua da Ponte, Ed. 23, Centro, Rio de Janeiro/RJ \\ marcosdossantos_doutorado_uff@yahoo.com.br \\ Carlos Francisco Simões Gomes \\ Universidade Federal Fluminense (UFF) \\ Rua Passo da Pátria, 156 - 209, São Domingos, Niterói - RJ \\ cfsg1@bol.com.br
}

\begin{abstract}
RESUMO
O presente estudo destacou a importância da utilização de métodos multicritério para auxiliar a tomada de decisão. Neste sentido, realizou-se um estudo de caso em uma empresa do ramo de iluminação decorativa objetivando ranquear três alternativas de lâmpada de filamento a luz de cinco critérios. Para tanto, foi utilizado o método SAPEVO-M, cujos cálculos matemáticos foram realizados por meio do software SAPEVO Web, disponível gratuitamente no site www.sapevoweb.com. Os julgamentos foram realizados por dois decisores, que avaliaram as preferências dos critérios e alternativas. Os resultados colaboraram para a escolha da melhor alternativa de lâmpada de filamento, que foi a de LED Ambar, a qual será adquirida para compor os recursos materiais da empresa em tela. Sendo assim, destaca-se que este método contribuiu para a tomada de decisão, atingindo o objetivo da empresa e demonstrando uma aplicação para o mesmo.
\end{abstract}

Palavra-chave: SAPEVO-M; SAPEVO Web; iluminação decorativa; Apoio multicritério a decisão; lâmpada de filamento. 


\begin{abstract}
The present study highlighted the importance of using multicriteria methods for auxiliary decision making. In this sense, a case study was carried out in a company of decorative lighting aiming to rank three alternatives of filament lamp under five criteria. For this purpose, the SAPEVO-M method was used, whose mathematical calculations were performed using the SAPEVO Web software, available for free at www.sapevoweb.com. The judgments were made by two decision makers, who evaluated the preferences of the criteria and alternatives. The results contributed to the choice of the best filament lamp alternative, which was Ambar LED, which will be acquired to compose the material resources of the company in question. Thus, it is noteworthy that this method contributed to decision making, achieving the company's objective and demonstrating an application for it.
\end{abstract}

Keywords: SAPEVO-M; SAPEVO Web; decorative illumination; Multicriteria Decision Aid; filament lamp.

\title{
Como Citar:
}

ALVES, Camila Guimarães Monteiro de Freitas; SANTOS, Marcos dos; GOMES, Carlos Francisco Simões. Seleção de uma lâmpada de filamento em uma empresa de iluminação decorativa: Uma aplicação do método SAPEVO-M. In: SIMPÓSIO DE PESQUISA OPERACIONAL E LOGÍSTICA DA MARINHA, 19., 2019, Rio de Janeiro, RJ. Anais [...]. Rio de Janeiro: Centro de Análises de Sistemas Navais, 2019.

\section{INTRODUÇÃO}

A tomada de decisão está presente no dia-a-dia das organizações, tornando o processo decisório cada vez mais importante no intuito de buscar as melhores alternativas.

Atualmente, o mundo dos negócios muda a uma velocidade cada vez maior e, diante deste cenário, é necessária a assertividade na tomada de decisão para a sobrevivência no mercado competitivo (OSHIRO; CRNKOVIC; SANTOS, 2015).

A tomada de decisão é dificultada por abarcar dados imprecisos ou incompletos, múltiplos critérios e vários decisores. Nesse contexto, surgem os métodos multicritérios para apoiar a tomada de decisão, visto que possibilita comparar alternativas diante da avaliação de critérios.

Considerando a dificuldade de encontrar soluções que sejam simultaneamente "ótimas" sob todos os pontos que estão sendo analisados, os métodos multicritério permitem a seleção da melhor alternativa possível.

Os métodos multicritérios podem ser aplicados para auxiliar a tomada de decisão em empresas de qualquer ramo e porte. Sendo assim, neste estudo, foi utilizado o método SAPEVO-M para auxiliar na escolha de uma lâmpada de filamento em uma empresa de iluminação decorativa localizada na região Sul Fluminense, estado do Rio de Janeiro. A partir dos resultados obtidos com a aplicação do método torna-se mais fácil a escolha da alternativa a luz dos critérios determinados.

Após esta introdução, segue-se a descrição do problema. Em seguida, abordam-se os conceitos principais relacionados aos métodos de apoio à decisão e, mais especificamente, ao método SAPEVO-M. Posteriormente é relatado um estudo de caso no qual se aplica o método SAPEVO-M para escolha de uma lâmpada de filamento e seus resultados. Por último, têm-se as conclusões do estudo. 


\section{DESCRIÇÃO DO PROBLEMA}

A iluminação na decoração de um evento seja casamento, formatura, aniversário de 15 anos ou infantil, faz toda diferença. O tipo de iluminação usada pode transformar o ambiente, valorizando todos os aspectos do evento que foram pensados e atingindo ou superando as expectativas que são criadas.

Existem diversos pontos que demonstram a importância da escolha adequada quanto ao tipo de iluminação a ser empregada. Um destes pontos diz respeito à interferência da iluminação na qualidade das fotos. Outro aspecto a ser considerado é que a iluminação ajuda a criar o clima desejado para cada momento. A iluminação também ajuda a realçar os ambientes. Uma iluminação eficaz não só auxilia a realçar a decoração escolhida como também a complementa. Pode-se citar também que a iluminação pode ser utilizada para sinalizar espaços ou indicar caminhos. Uma característica da iluminação é tornar o ambiente mais funcional.

Muitas são as variáveis a serem analisadas ao se optar pela iluminação mais adequada a ser utilizada, o que demanda assertividade no momento da escolha de compra pelo artefato ideal. Para uma empresa de iluminação, adquirir os artefatos ideais a serem utilizados nos eventos para os quais a mesma será contratada é de grande importância tendo em vista a necessidade de otimização dos recursos materiais e financeiros.

Para entender a necessidade de aplicação da análise multicritério, primeiramente se identifica o cenário do problema que, neste caso específico, envolve múltiplos critérios que possuem níveis de importância diferenciados, unidades de medidas variadas, dificuldade para tratar a subjetividade, e existência de mais de um decisor. Os critérios elencados e as alternativas podem ser visualizados na Figura 1.

Figura 1 - Critérios e alternativas do problema

\begin{tabular}{|l|l|l|}
\hline $\begin{array}{c}\text { Lâmpada } \\
\text { de } \\
\text { Filamento }\end{array}$ \\
Pluxo luminoso
\end{tabular}

\section{REVISÃO BIBLIOGRÁFICA}

\subsection{APOIO MULTICRITÉRIO A DECISÃO}

O apoio multicritério à decisão, em inglês Multiple-Criteria Decision Making (MCDM) ou multiple criteria decision aid (MCDA), refere-se ao ramo da tomada de decisão que lida com a presença de diferentes critérios sob situação de conflito.

Segundo Clintworth, Boulougouris e Lee (2017) as abordagens tradicionais de MCDA são usadas para apoiar os tomadores de decisão em contextos complexos, conflitantes e incertos. Doan e De Semet (2018) destacam que a maioria dos problemas de 
decisão estratégica envolve a otimização simultânea de vários critérios conflitantes.

Nesse contexto, são empregados métodos de apoio à decisão que possibilitam avaliar problemas à luz de uma perspectiva múltipla - tais como os métodos de auxílio multicritério à decisão (AMD) (NEVES; PEREIRA; COSTA, 2015).

Existem diversos métodos multicritério que auxiliam a tomada de decisão, os quais são utilizados para estruturar problemas tanto de instituições públicas quanto privadas, envolvendo decisões baseadas em múltiplos critérios e uma complexidade e diversidade de alternativas (SANTOS; GOMES; LIMA, 2019).

De acordo com Santos et al (2017) os métodos multicritério de apoio à decisão levam em consideração tanto um caráter científico quanto subjetivo, englobando características quantitativas e qualitativas.

Os métodos multicritério pressupõem um diálogo com o tomador de decisão e visa recomendar a melhor solução com base nas preferências deste. O processo de auxílio à decisão multicritério é composto pelas seguintes etapas: estruturação do problema de decisão, modelagem de preferências, agregação, exploração e recomendação (FRINI, 2017).

Um ponto destacado por Carvalho, Poleto e Costa (2015) versa sobre a importância da definição da problemática de decisão que irá auxiliar na deliberação do método a ser empregado para o apoio à decisão. As alternativas são avaliadas segundo os múltiplos critérios definidos e cada critério também será avaliado pelo decisor.

Muitas classificações são utilizadas para designar os métodos multicritério de apoio à decisão. Dentre estas, a de maior repercussão é a que subdivide os métodos da Escola Americana e os métodos da Escola Francesa, também designada Escola Europeia. Entretanto, atualmente, ressalta-se a aplicação de métodos compensatórios e nãocompensatórios com problemáticas distintas (FERREIRA et al, 2008).

Com relação à problemática, Roy (1996) descreveu as seguintes tipologias: Problemática P. $\alpha$ ou de Escolha, Problemática P. $\beta$ ou de Classificação, Problemática P.ץ ou de Ordenação, Problemática P. $\delta$ ou de Descrição.

Corroborando as problemáticas descritas por Roy (1996), Luiz et al (2017) destacaram as seguintes problemáticas: a) encontrar a melhor alternativa; b) agrupar as alternativas dentro de classes definidas, c) ranquear as alternativas e d) descrever como cada alternativa atende a todos os critérios simultaneamente, conforme proposto.

Já Doumpos e Figueira (2019) relatam que os métodos de ordenação podem ser classificados em métodos cardinal ou ordinal.

\subsection{SAPEVO-M}

O método SAPEVO (Simple Aggregation of Preferences Expressed by Ordinal Vectors) é um método multicritério não compensatório de ranqueamento de alternativas que pode utilizar variáveis qualitativas e quantitativas, no qual se deve assumir que para um determinado cenário de classificação multicritério apenas os critérios essenciais devem ser utilizados (GOMES; MURY; GOMES, 1997).

Já o método SAPEVO-M representa uma nova versão do método SAPEVO, e considera múltiplos decisores. O SAPEVO-M introduziu um processo de normalização das matrizes de avaliação, o que permitiu aumentar sua consistência (SANTOS et al, 2019).

Segundo os autores, o método SAPEVO-M consiste em dois processos. Primeiramente, é feita a transformação da preferência ordinal dos critérios, gerando o vetor de peso dos critérios. Posteriormente, é realizada a transformação ordinal da preferência entre alternativas em relação a um conjunto de critérios, gerando uma matriz. Em seguida, é levado em consideração a matriz de alternativas e o vetor de critérios. 


\section{ESTUDO DE CASO}

A empresa analisada neste estudo é uma empresa de iluminação decorativa localizada na cidade de Volta Redonda, região Sul Fluminense do estado do Rio de Janeiro. Esta empresa encontra-se no mercado há mais de 5 anos e trabalha de forma a valorizar todos os detalhes da decoração e arquitetura do ambiente de diversos tipos de eventos: casamento, 15 anos, formatura, festa infantil, confraternização empresarial e outros.

Foram entrevistados o dono da empresa e um funcionário para definição dos critérios e realização dos julgamentos.

Os critérios utilizados foram custo unitário, temperatura de cor, fluxo luminoso, potência e vida útil.

O custo unitário representa o preço de custo pelo qual as lâmpadas serão adquiridas.

A temperatura de cor está relacionada à aparência de cor da luz emitida pela lâmpada. Quanto mais alta a temperatura de cor, mais clara é a tonalidade de cor. A característica de uma luz ser quente ou fria diz respeito à tonalidade de cor que a mesma irradia ao ambiente e não ao calor físico da lâmpada. Em uma iluminação decorativa, a temperatura de cor influencia diretamente no ambiente e deve ser escolhida de acordo com sua finalidade.

Quanto ao fluxo luminoso, este versa sobra a quantidade de luz emitida por uma lâmpada em todas as direções, isto é, o quanto uma lâmpada ilumina um ambiente. O fluxo luminoso é medido em Lúmen e quanto maior esse número, mais luz a lâmpada emite. É o fluxo luminoso que influencia a percepção de melhor iluminação no ambiente, pois é ele que se aplica ao estímulo visual, e não a potência, que representa a quantidade de energia gasta para realizar esse trabalho. A potência, cuja unidade de medida é o watt, diz respeito ao consumo de energia, e não a emissão de luz.

Já a vida útil é a expectativa de durabilidade de uma fonte luminosa.

Neste estudo, foram analisadas três alternativas de lâmpada de filamento: Filamento de Carbono modelo G95, filamento de LED modelo G125 e filamento de LED Ambar modelo G80. As lâmpadas de filamento são muito utilizadas para fins decorativos. Este tipo de lâmpada forma um desenho de linhas com seu filamento exposto dentro do bulbo de vidro. Estas lâmpadas podem ser utilizadas em abajures, pendentes e luminárias, sozinhas ou em conjunto.

As lâmpadas de filamento de carbono foram recriadas a partir do conceito do filamento de carbono das primeiras lâmpadas elétricas inventadas no final dos anos 1800 que possuíam grandes anéis de filamento de carbono que produziam luz única e quente. As novas lâmpadas de filamento de carbono possuem uma durabilidade maior. Uma lâmpada de filamento de carbono tem mais filamentos. Entretanto, sua iluminação é de menor intensidade quando comparada às demais lâmpadas de filamento.

As lâmpadas de filamento de LED possuem alta eficiência de diodos emissores de luz. A tecnologia LED transforma a energia em iluminação ao invés de calor e tem maior vida útil.

A lâmpada de filamento de LED com revestimento de âmbar proporciona economia imediata produzindo maior luminosidade utilizando menos energia, quando comparada com as lâmpadas com filamento de carbono.

Os valores relacionados aos critérios em relação a cada alternativa podem ser observados na Tabela 1. 
Tabela 1 - Critérios x Alternativas

\begin{tabular}{|l|c|c|c|c|c|}
\hline \multicolumn{1}{|c|}{ Alternativas } & $\begin{array}{c}\text { Custo } \\
\text { unitário }\end{array}$ & Potência & $\begin{array}{c}\text { Temperatura } \\
\text { da Cor (kelvin) }\end{array}$ & $\begin{array}{c}\text { Fluxo } \\
\text { Luminoso } \\
\text { (lumens) }\end{array}$ & $\begin{array}{c}\text { Vida útil } \\
\text { (horas) }\end{array}$ \\
\hline $\begin{array}{l}\text { Lâmpada Filamento } \\
\text { de Carbono } \\
\text { Modelo g95 }\end{array}$ & $\mathrm{R} \$ 21,99$ & $40 \mathrm{w}$ & 2.200 & 100 & 2.000 \\
\hline $\begin{array}{l}\text { Lâmpada de } \\
\text { Filamento Led } \\
\text { Modelo G125 }\end{array}$ & $\mathrm{R} \$ 29,90$ & $3,2 \mathrm{w}$ & 3.000 & 320 & 40.000 \\
\hline $\begin{array}{l}\text { Lâmpada Filamento } \\
\text { de LED Ambar } \\
\text { Modelo G80 }\end{array}$ & $\mathrm{R} \$ 25,00$ & $8 \mathrm{w}$ & 2.300 & 750 & 20.000 \\
\hline
\end{tabular}

\subsection{APLICAÇÃO DO MÉTODO SAPEVO-M}

A aplicação do modelo matemático SAPEVO-M se deu por meio do software SAPEVO Web, desenvolvido pelos pesquisadores do Instituto Militar de Engenharia, disponível no site www.sapevoweb.com.

Em um primeiro momento, os decisores, que foram o dono da empresa e um funcionário, foram cadastrados no sistema, conforme observado na Figura 2.

Figura 2 - Cadastro dos decisores no software SAPEVO Web

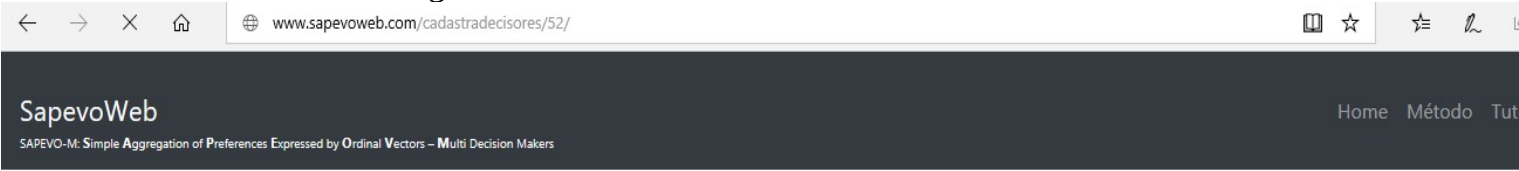

\section{Projeto lluminação}

\section{Cadastrar Decisor}

Nome: Funcionário $\quad$ Cadastrar Finalizar

Decisores

Dono da empresa 
3.

Em seguida, foram cadastradas as alternativas, o que pode ser visualizado na Figura

Figura 3 - Cadastro das alternativas no software SAPEVO Web

(1) www.sapevoweb.com/cadastraalternativas/52/

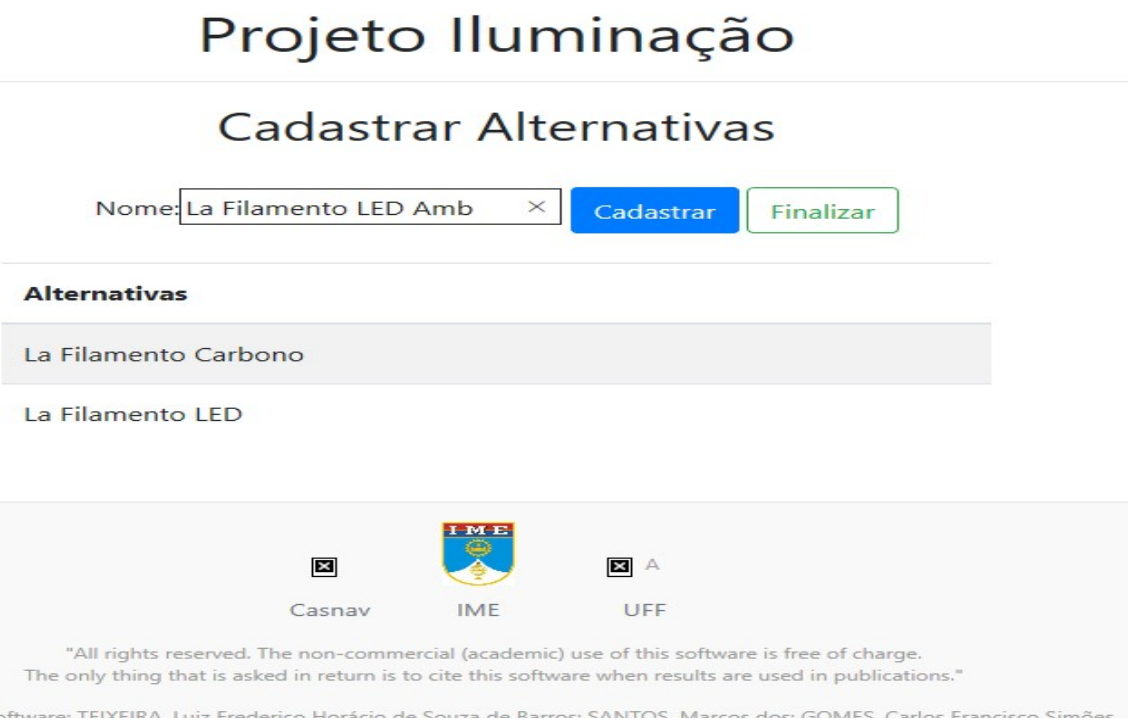

Posteriormente, os critérios também foram inseridos no sistema, de acordo com o demonstrado na Figura 4.

Figura 4 - Cadastro dos critérios no software SAPEVO Web

(1) www.sapevoweb.com/cadastracriterios/52/

\section{Projeto Iluminação}

\section{Cadastrar Critério}

Nome: Vida útil $\quad \times \quad$ Cadastrar Finalizar

Critérios

Custo unitário

Potência

Temperatura da Cor

Fluxo Luminoso

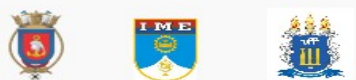

Os decisores analisaram os critérios e alternativas de acordo com suas preferências, em uma escala ordinal. Posteriormente, a cada critério foram atribuídos pesos, conforme Tabela 2, de acordo com os julgamentos feitos pelos decisores. 
Tabela 2 - Escala de Pesos

\begin{tabular}{|c|l|c|}
\hline $\begin{array}{c}\text { Escala 1 } \\
\text { (Símbolo) }\end{array}$ & Escala 1 (Variável / expressão linguística correspondente) & Escala 2 \\
\hline$<<\ll 1$ & Absolutamente pior / Absolutamente menos importante & -3 \\
\hline$<« 1$ & Muito pior / Muito menos importante & -2 \\
\hline$« 1$ & Pior / Menos importante & -1 \\
\hline 1 & Igual pu equivalente / Tão importante quanto & 0 \\
\hline$» 1$ & Melhor / Mais importante & 1 \\
\hline$>» 1$ & Muito melhor / Muito mais importante & 2 \\
\hline$>>» 1$ & Absolutamente melhor /Absolutamente mais importante & 3 \\
\hline
\end{tabular}

Fonte: Gomes, Mury e Gomes (1997)

Com todas as etapas cadastradas, iniciaram-se os julgamentos do dono da empresa quanto aos critérios, que podem ser observados na Figura 5.

Figura 5 - Julgamentos dos critérios - Dono da empresa

www.sapevoweb.com/avaliarcriterios/52/

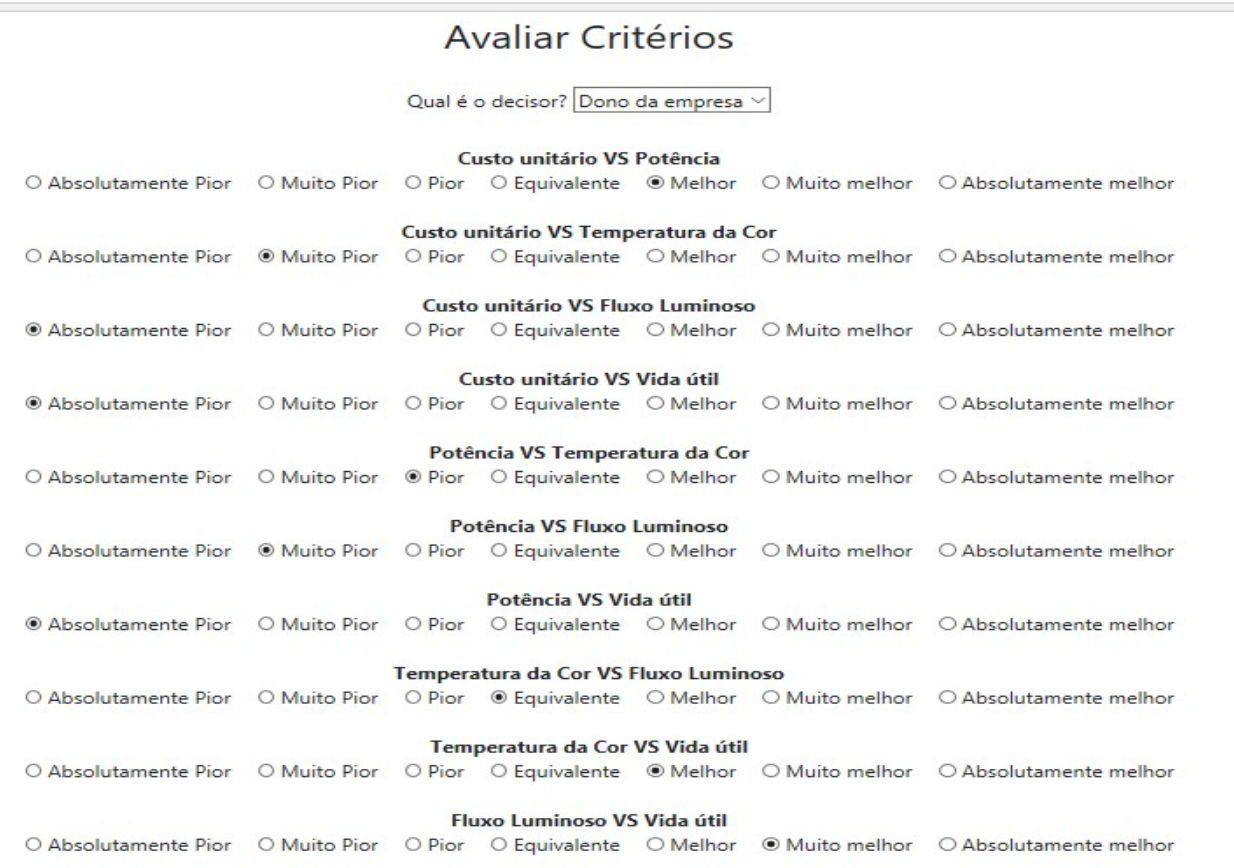

Já os julgamentos do funcionário em relação aos critérios estão descritos na Figura

6. 
Figura 6 - Julgamentos dos critérios - Funcionário

www.sapevoweb.com/avaliarcriterios/52/

\begin{tabular}{|c|c|c|c|c|c|c|}
\hline \multicolumn{7}{|c|}{ Avaliar Critérios } \\
\hline \multicolumn{7}{|c|}{ Qual é o decisor? Funcionário } \\
\hline \multicolumn{7}{|c|}{ Custo unitário VS Potência } \\
\hline O Absolutamente Pior & Muito Pior & O Pior & Equivalente & ○ Melhor & Muito melhor & Absolutamente melhor \\
\hline \multicolumn{7}{|c|}{ Custo unitário VS Temperatura da Cor } \\
\hline ( ) Absolutamente Pior & Muito Pior & O Pior & O Equivalente & O Melhor & Muito melhor & Absolutamente melhor \\
\hline \multicolumn{7}{|c|}{ Custo unitário VS Fluxo Luminoso } \\
\hline - Absolutamente Pior & O Muito Pior & O Pior & O Equivalente & Melhor & Muito melhor & Absolutamente melhor \\
\hline \multicolumn{7}{|c|}{ Custo unitário VS Vida útil } \\
\hline O Absolutamente Pior & - Muito Pior & O Pior & Equivalente & OMelhor & Muito melhor & Absolutamente melhor \\
\hline \multicolumn{7}{|c|}{ Potência VS Temperatura da Cor } \\
\hline Absolutamente Pior & - Muito Pior & O Pior & Equivalente & OMelhor & Muito melhor & Absolutamente melhor \\
\hline \multicolumn{7}{|c|}{ Potência VS Fluxo Luminoso } \\
\hline (1) Absolutamente Pior & Muito Pior & O Pior & Equivalente & Melhor & Muito melhor & Absolutamente melhor \\
\hline \multicolumn{7}{|c|}{ Potência VS Vida útil } \\
\hline O Absolutamente Pior & - Muito Pior & O Pior & Equivalente & OMelhor & Muito melhor & Absolutamente melhor \\
\hline \multicolumn{7}{|c|}{ Temperatura da Cor VS Fluxo Luminoso } \\
\hline Absolutamente Pior & O Muito Pior & O Pior & (- Equivalente & Melhor & Muito melhor & Absolutamente melhor \\
\hline \multicolumn{7}{|c|}{ Temperatura da Cor VS Vida útil } \\
\hline O Absolutamente Pior & O Muito Pior & O Pior & O Equivalente & Melhor & - Muito melhor & O Absolutamente melhor \\
\hline \multicolumn{7}{|c|}{ Fluxo Luminoso VS Vida útil } \\
\hline O Absolutamente Pior & O Muito Pior & O Pior & Equivalente & Melhor & Muito melhor & - Absolutamente melhor \\
\hline
\end{tabular}

Após o julgamento de preferências dos critérios, partiu-se para o julgamento das alternativas. Na Figura 7, observa-se a avaliação do critério Custo Unitário para o decisor Dono da empresa.

Figura 7 - Julgamento do Dono da empresa para o critério Custo unitário

Critério - Custo unitário - ID $=>130$
La Filamento Carbono VS La Filamento LED
O Absolutamente Pior OMuito Pior O Pior O Equivalente O Melhor $\odot$ Muito melhor $\bigcirc$ Absolutamente melhor
La Filamento Carbono VS La Filamento LED Amb
OAbsolutamente Pior OMuito Pior OPior O Equivalente $\odot$ Melhor $\bigcirc$ Muito melhor $\bigcirc$ Absolutamente melhor
La Filamento LED vS La Filamento LED Amb

Na Figura 8 têm-se o julgamento do Dono da empresa para o critério Potência em relação as três alternativas.

Figura 8 - Julgamento do Dono da empresa para o critério Potência

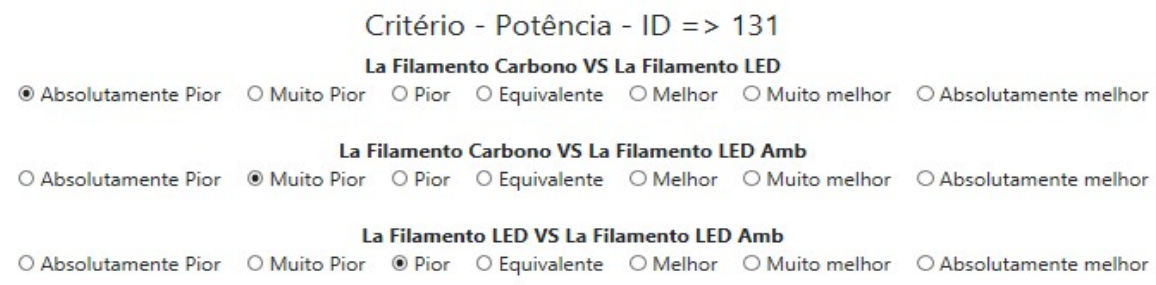


Já na Figura 9 encontra-se o julgamento do Dono da empresa para o critério temperatura da cor em relação as três alternativas.

Figura 9 - Julgamento do Dono da empresa para o critério Temperatura da cor

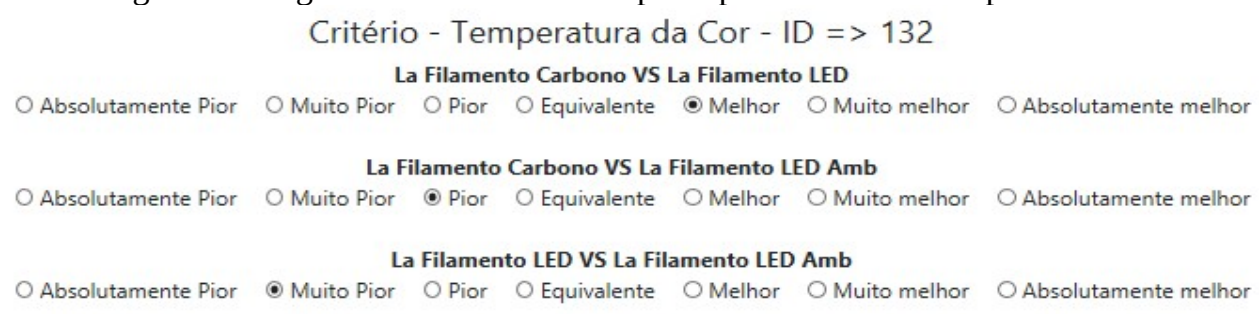

Na Figura 10, observa-se a avaliação do critério Fluxo luminoso para o decisor Dono da empresa.

Figura 10 - Julgamento do Dono da empresa para o critério Fluxo luminoso

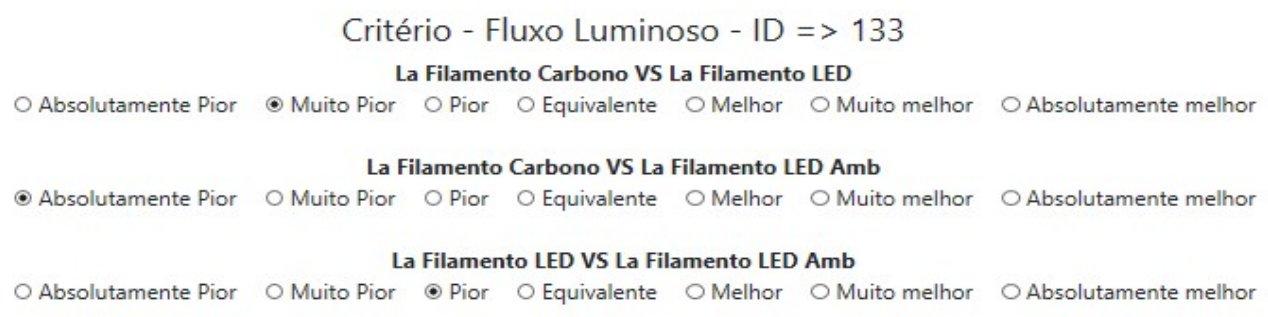

Já na Figura 11 visualiza-se o julgamento do Dono da empresa para o vida útil em relação as três alternativas.

Figura 11 - Julgamento do Dono da empresa para o critério Vida útil

\begin{tabular}{|c|c|c|c|c|}
\hline \multicolumn{5}{|c|}{ Critério - Vida útil - ID => 134} \\
\hline \multicolumn{5}{|c|}{ La Filamento Carbono VS La Filamento LED } \\
\hline (- Absolutamente Pior & O Muito Pior O Pior & Equivalente $O$ Melhor & Muito melhor & Absolutamente melhor \\
\hline \multicolumn{5}{|c|}{ La Filamento Carbono VS La Filamento LED Amb } \\
\hline O Absolutamente Pior & (-) Muito Pior O Pior & O Equivalente $O$ Melhor & Muito melhor & A Absolutamente melhor \\
\hline \multicolumn{5}{|c|}{ La Filamento LED VS La Filamento LED Amb } \\
\hline Absolutamente Pior & O Muito Pior O Pior & Equivalente $O$ Melhor & (-) Muito melhor & Absolutamente melhor \\
\hline
\end{tabular}

Os julgamentos com relação as alternativas para cada critério também foram realizados pelo funcionário. O julgamento quanto ao critério custo unitário podem ser observados na Figura 12.

Figura 12 - Julgamento do funcionário para o critério Custo unitário

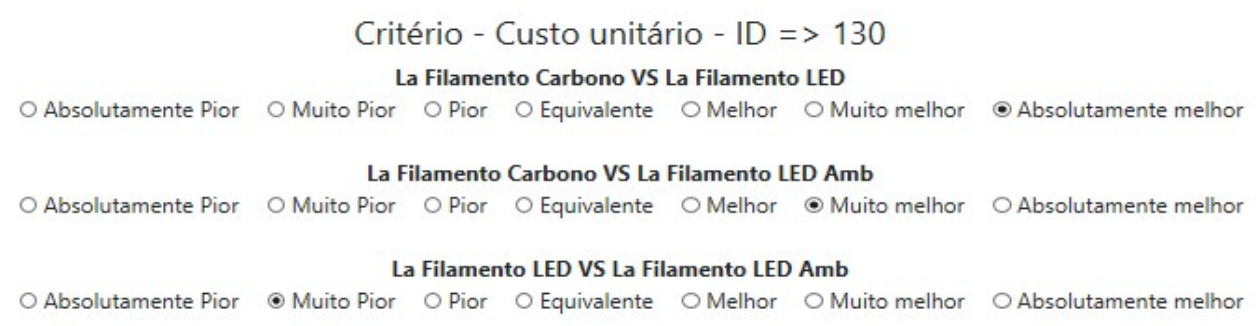

Na Figura 13 tem-se o julgamento do funcionário para o critério Potência em relação as três alternativas. 
Figura 13 - Julgamento do funcionário para o critério Potência

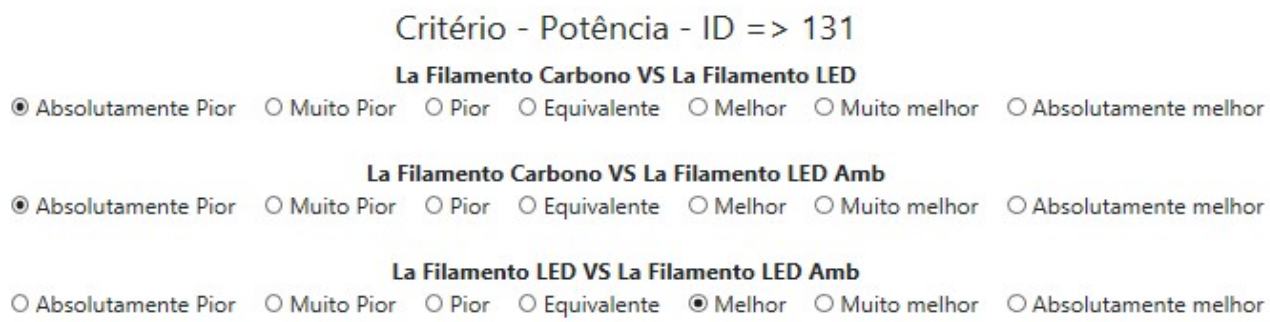

Já na Figura 14 visualiza-se o julgamento do funcionário para Temperatura da cor em relação as três alternativas.

Figura 14 - Julgamento do funcionário para o critério Temperatura da cor

Critério - Temperatura da Cor - ID => 132

La Filamento Carbono VS La Filamento LED

O Absolutamente Pior O Muito Pior O Pior O Equivalente $\odot$ Melhor O Muito melhor O Absolutamente melhor

La Filamento Carbono VS La Filamento LED Amb

O Absolutamente Pior O Muito Pior $\odot$ Pior O Equivalente OMelhor O Muito melhor O Absolutamente melhor

La Filamento LED VS La Filamento LED Amb

O Absolutamente Pior $\odot$ Muito Pior O Pior O Equivalente OMelhor O Muito melhor OAbsolutamente melhor funcionário.

Na Figura 15, destaca-se a avaliação do critério Fluxo luminoso para o decisor Figura 15 - Julgamento do funcionário para o critério Fluxo luminoso

Critério - Fluxo Luminoso - ID =>133

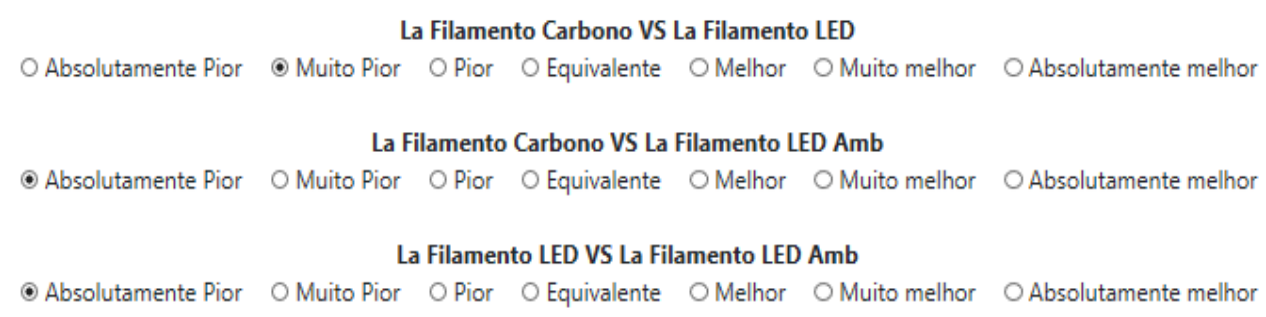

Já a comparação paritária das alternativas para o critério vida útil a partir do julgamento do funcionário pode ser observada na Figura 16.

Figura 16 - Julgamento do funcionário para o critério Vida útil

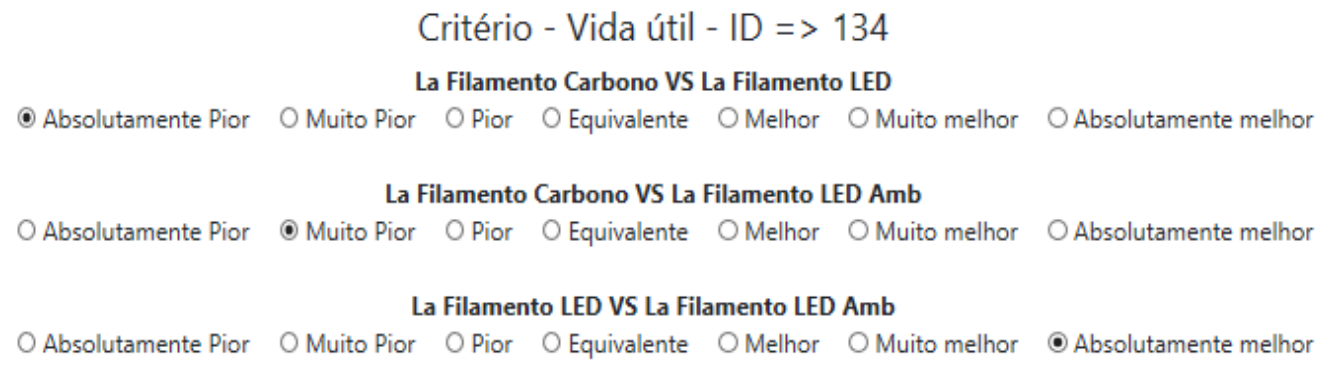




\section{RESULTADOS}

A partir da aplicação do Método SAPEVO-M pela ferramenta computacional SAPEVO Web, com os julgamentos dos critérios realizados pelos decisores, obteve-se os pesos observados na Figura 17.

O critério de maior peso foi Fluxo luminoso, seguido de temperatura da cor, vida útil, custo unitário e, por último, potência.

Figura 17 - Pesos dos critérios

\section{Pesos}

Critério - Custo unitário - 0.06596638655462185

Critério - Potência - 0.007731092436974791

Critério - Temperatura da Cor - 1.6680672268907561

Critério - Fluxo Luminoso - 2.0

Critério - Vida útil - 1.1260504201680672

A partir dos pesos dos critérios e dos julgamentos das alternativas, pode-se observar a ordenação das alternativas na Figura 18. Em primeiro lugar, encontra-se a lâmpada de filamento LED Ambar, seguida por lâmpada de filamento LED e, por último, filamento de Carbono.

Figura 18 - Ordenação das alternativas

$$
\begin{gathered}
\text { Ordenação } \\
1^{\circ} \text {-- La Filamento LED Amb -- } 8.388514896867838 \\
2^{\circ} \text {-- La Filamento LED -- } 4.327202699261523 \\
3^{\circ} \text {-- La Filamento Carbono -- } 1.7999999999999998
\end{gathered}
$$

\section{CONCLUSÃO}

A escolha adequada da lâmpada de filamento influencia na qualidade do serviço de iluminação decorativa que será prestado. Todos os critérios definidos são importantes para a escolha adequada da melhor alternativa e o método SAPEVO-M permite considera-los com suas devidas preferências nessa análise. Além disso, os recursos financeiros são limitados e as decisões devem ser acertadas para evitarem-se aquisições que não contemplem da melhor maneira possível os critérios em suas preferências.

Com a aplicação do Método SAPEVO-M facilitou-se o processo decisório e ampliou-se a assertividade na tomada de decisão. Este método pode ser utilizado para auxiliar a tomada de decisão tanto em grandes quanto pequenas empresas, públicas ou privadas, de todos os ramos, inclusive para operações de serviço.

Destaca-se que a ferramenta computacional SAPEVO Web, desenvolvida pelo 
Instituto Militar de Engenharia em cooperação com a Universidade Federal Fluminense, é de simples utilização e os resultados demonstrados de forma clara.

\section{REFERÊNCIAS BIBLIOGRÁFICAS}

[1] CARVAlHO, V. D. H.; POLETO, T.; Silva, L. C.; Costa, A. P. C. C. Abordagem multicritério de apoio a decisões estrategicamente sustentáveis nas organizações. Revista Produção Online, v.15, n. 3, p. 925-947, 2015.

[2] CLINTWORTH, M.; BOULOUGOURIS, E.; LEE, B. S. Combined MCDA e CBA in the assessment of maritime projects financed by the European Investiment Bank. Maritime Economics \& Logistics, p. 1-19, 2017.

[3] DOAN, N. A. V.; DE SEMET, Y. An alternative weight sensitivity analysis for PROMETHEE II rankings. Omega, v. 80, p. 166 - 174, 2018.

[4] DOUMPOS, M.; FIGUEIRA, J. R. A multicriteria outranking approach for modeling corporate credit ratings: an application of the Electre Tri-nC method. Omega, v. 82, p. 166 - 180, 2019.

[5] FERREIRA, M. M. G. et al. Escolha dos métodos de multicritério a tomada de decisão com o auxílio de um sistema especialista formulado a partir de um fluxograma. Brazilian Applied Science Review, v. 2, n. 5, p. 1593-1608, 2018.

[6] FRINI, A. A Multicriteria Intelligence Aid Methodology Using MCDA, Artificial Intelligence, and Fuzzy Sets Theory. Mathematical Problems in Engineering, p. 1 10, 2017.

[7] GOMES, L. F. A. M.; MURY, A. R.; GOMES, C. F. S. Multicriteria ranking with ordinal data. SAMS, vol 27, p. 139 - 145, 1997.

[8] LUIZ, Washington. et al. Inserção de critérios de sustentabilidade na matriz de decisão altera alternativa de aquisição de bens em uma instituição pública. International Scientific Journal, v. 12, n. 2, p. 148 - 172, 2017.

[9] OSHIRO, Sonia.; CRNKOVIC, Luciana Helena.; SANTOS, Fernando César Almada. O desafio de integrar cultura organizacional e a gestão da produção. RAU - Revista de Administração da UNIMEP, v. 3, n. 2, 2015.

[10] NEVES, R. B.; PEREIRA, V.; COSTA, H. G. Auxílio multicritério à decisão aplicado ao planejamento e gestão na indústria de petróleo e gás. Production, v. 25, n. 1, p. 4353, 2015.

[11] ROY, B. Multicriteria methodology for decision aiding. Kluwer Academic Publishers, 1996.

[12] Santos, Marcos dos; Rodriguez, T. O, Quintal, R. S., Dias, F. C. e Reis, M. F. Emprego de Métodos Multicritério para apoio à decisão em empreendimento turísticos: o caso do Hostel Ocean inn Rio. CULTUR: Revista de Cultura e Turismo, v. 11, p. 87-107, 2017.

[13] GRECO, Tomás; SANTOS, Marcos dos; GOMES, Carlos Francisco Simões; LIMA, Angélica Rodrigues de. Escolha de um Navio de Desembarque de Tropa para a Armada Argentina por Meio do Método SAPEVO com Múltiplos Decisores (SAPEVO M). In: Simpósio de Aplicações Operacionais em Áreas de Defesa, 22, 2019, São Paulo. Anais. São Paulo: SIGE, 2019. 
[14] SANTOS, Marcos dos; CUPERTINO, Nathália de Farias; GOMES, Carlos Francisco Simões; LIMA, Angélica Rodrigues; REIS, Marcone Freitas. Seleção de pessoal para uma empresa de gases medicinais e industriais a partir do método SAPEVO-M. In: Simpósio Nacional de Engenharia de Produção, 2, 2019, Mato Grosso do Sul. Anais... Mato Grosso do Sul: SINEP: 2019 